\title{
La dimension critique de l'éducation relative à l'environnement dans un pays en développement : réflexions issues d'une expérience sénégalaise
}

Michèle Berthelot

\section{(Q) OpenEdition}

\section{Journals}

Édition électronique

URL : http://journals.openedition.org/ere/3209

DOI : $10.4000 /$ ere.3209

ISSN : 2561-2271

Éditeur

Centr'ERE

Référence électronique

Michèle Berthelot, «La dimension critique de l'éducation relative à l'environnement dans un pays en développement : réflexions issues d'une expérience sénégalaise », Éducation relative à l'environnement [En ligne], Volume 7 | 2008, mis en ligne le 20 septembre 2008, consulté le 21 février 2020. URL : http://journals.openedition.org/ere/3209; DOI : 10.4000/ere.3209 


\title{
La dimension critique de l'éducation relative à l'environnement dans un pays en développement : réflexions issues d'une expérience sénégalaise
}

\author{
Michèle Berthelot
}

1 De nombreux auteurs ont mis en évidence que les apports des projets d'aide au développement sont le plus souvent peu durables et que les activités qui s'y rattachent ne survivent généralement pas à l'arrêt du financement des bailleurs de fonds (Latouche, 1998 ; Naudet, 1999 ; Traoré, 1999 ; Smith, 2003). À l'issue d'une recherche doctorale sur le sujet ${ }^{1}$, j'ai pu constater que l'implantation du Programme de formationinformation pour l'environnement (PFIE) au Sénégal n'échappait pas complètement à cette logique.

2 Par ailleurs, au-delà du degré de pérennisation de ce programme, il paraît fondamental de s'interroger sur la teneur de la proposition d'éducation relative à l'environnement (ERE) qu'il portait et sur la nature des pratiques auxquelles elle a donné lieu dans les écoles. On comprendra aisément l'intérêt d'une telle analyse. À lui seul, un haut degré de pérennisation ne suffit pas à assurer la réussite d'un projet d'ERE. Encore faut-il que la proposition d'ERE qu'il porte soit riche, tant sur le plan des contenus environnementaux que des approches pédagogiques, et que ses aspects essentiels survivent aux conditions de sa mise en œuvre.

3 L'étude dont les résultats sont rapportés dans le présent article visait à caractériser, dans une perspective critique, la proposition formelle d'éducation relative à l'environnement (ERE) portée par le PFIE sénégalais et ce qu'elle est devenue à l'issue de sa mise en œuvre dans un certain nombre d'écoles ciblées.

4 Après une brève présentation du PFIE et des outils conceptuels et méthodologiques qui ont servi à sa caractérisation, les principaux résultats de l'analyse à laquelle il a donné lieu sont présentés. L'analyse s'intéresse, d'une part, au projet « initial », c'est-à-dire à ses objectifs, à sa stratégie d'implantation ainsi qu'au programme d'éducation 
environnementale (EE) et au manuel scolaire qui en constituaient la pierre angulaire et, d'autre part, au projet "effectif», c'est-à-dire tel qu'il s'est concrétisé dans les représentations et les pratiques des acteurs qui ont participé à sa mise en œuvre.

Par la suite, une mise en relation des constats dégagés avec les éléments du contexte sénégalais permettra de mieux saisir l'aspect critique que renferment certaines des pratiques d'ERE rapportées.

\section{L'expérience du PFIE sénégalais}

6 Le Programme de formation-information pour l'environnement (PFIE) est le volet relatif à l'enseignement primaire du Programme sahélien d'éducation (PSE) adopté en 1988 par le Comité Inter-États de Lutte contre la Sécheresse au Sahel (CILSS). Le CILSS comptait ainsi inverser la tendance à la fragilisation des économies du Sahel et à la paupérisation croissante de ses populations, induites par la détérioration de l'environnement au cours des dernières décennies.

7 Le PFIE, financé par l'Union européenne au titre des aides non remboursables, a été implanté au cours de la décennie 1990-2000 dans les neuf États membres du CILSS : Burkina Faso, Cap-Vert, Gambie, Guinée-Bissau, Mali, Mauritanie, Niger, Sénégal et Tchad.

8 Au Sénégal, trois objectifs généraux ont été assignés au PFIE (PFIE/Sénégal, 1999, p. 1) :

a. améliorer la qualité et l'efficacité de l'enseignement dispensé à l'école primaire ;

b. renforcer les capacités locales pour la gestion des ressources naturelles;

c. sensibiliser les populations sahéliennes aux problèmes de l'environnement à travers l'école.

9 Le Sénégal a implanté le PFIE à une échelle relativement étendue: plus de 20 circonscriptions scolaires ont été couvertes; plus de 5000 maitres et de 150000 élèves ont été formés; un vaste éventail de démarches et d'outils pédagogiques à l'intention des divers acteurs engagés en ERE a été développé. Le 30 juin 2001, le PFIE sénégalais a clôturé ses activités, après une phase de transition qui devait permettre de mettre en place des plans d'accompagnement en vue d'intégrer l'éducation environnementale à l'éducation de base des élèves de l'enseignement primaire. Au moment où les données de l'étude dont il est ici question ont été recueillies, entre 2002 et 2005, cette intégration n'avait pas encore eu lieu puisque le nouveau curriculum de l'enseignement primaire, outil privilégié de cette intégration, était en cours d'élaboration. Cependant, malgré l'arrêt formel du projet et le silence du curriculum officiel en matière d'éducation environnementale, les intervenants scolaires des écoles qui avaient participé à l'implantation du PFIE continuaient, à des degrés divers, de mettre en œuvre le PFIE.

\section{Des outils conceptuels et méthodologiques pour caractériser la proposition d'ERE du PFIE sénégalais}

Pour caractériser, dans une perspective critique, la proposition d'ERE portée initialement par le PFIE sénégalais et sa traduction effective dans les écoles participantes, il m'a paru intéressant de les mettre en relation avec des références connues du domaine de l'ERE, non pas pour évaluer l'ERE issue du PFIE à l'aune de 
prescriptions internationales, mais plutôt pour en cerner le positionnement par rapport à des textes fondateurs connus de tous.

11 À cet égard, les cinq axes de développement de l'ERE définis à l'issue de la Conférence de Tbilissi (UNESCO/PNUE, 1978, p. 30) constituent des points de repère utiles pour apprécier l'ampleur des finalités de la proposition d'ERE du PFIE : 1) prise de conscience de l'environnement et des problèmes qui y sont reliés ; 2) connaissances ; 3) état d'esprit ; 4) compétences de résolution de problèmes ; 5) participation individuelle et collective à la résolution des problèmes environnementaux.

12 Considérant qu'une analyse plus approfondie s'imposait, en particulier sur le plan épistémologique, j'ai aussi tenté de situer le PFIE par rapport aux principaux paradigmes en ERE (positiviste, interprétatif et sociocritique), tels qu'ils ont été définis par Ian Robottom et Paul Hart (1993, p. 26-27). Cette typologie, malgré ses limites, m’a fourni une grille de lecture pour orienter mon questionnement.

13 Enfin, en prenant appui sur les travaux de Lucie Sauvé ${ }^{2}$,je me suis aussi attachée à caractériser les représentations de l'environnement, de l'éducation et de l'éducation relative à l'environnement portées par le PFIE sénégalais de même que les dimensions éducationnelles et les approches pédagogiques privilégiées.

14 L'approche méthodologique retenue fait appel aux trois stratégies de collecte de données couramment utilisées en recherche qualitative : l'analyse documentaire, les entretiens et les observations. Le matériel documentaire analysé est constitué des documents de présentation et des bilans du projet produits par la Coordination nationale sénégalaise ainsi que du programme d'éducation environnementale et du manuel scolaire du 3e cycle du primaire produits par l'équipe du PFIE/Sénégal. Dix-huit entretiens semi-dirigés - 13 individuels et 6 de groupe - ont été réalisés avec 47 acteurs engagés à divers titres dans le PFIE (promoteurs-coordonnateurs du projet, partenaires, représentants des instances centrales et départementales du ministère de l'Éducation, intervenants des écoles). Chaque entretien a fait l'objet d'une transcription intégrale, qui a servi de base à une analyse de contenu. Enfin, des observations de "leçons d'ERE » ont été réalisées dans deux classes du primaire, l'une de $2 \mathrm{e}$ année et l'autre de $5 \mathrm{e}$ année, dans une école qui avait participé au PFIE 3 .

\section{Regard critique sur la proposition d'ERE du PFIE sénégalais}

15 À l'issue de l'analyse des données, comment peut-on caractériser le PFIE, tant en ce qui a trait aux objectifs et à la stratégie d'implantation adoptés, aux représentations de l'environnement, de l'éducation et de l'éducation relative à l'environnement privilégiées qu'aux pratiques d'ERE promues? Quel sort la proposition initiale du PFIE a-t-elle connu dans la réalité des écoles et des classes?

\section{Un projet ambitieux confronté à des traditions solidement ancrées}

\section{Des objectifs et une stratégie marqués par un désir d'innover}

Mise à part une certaine propension à vouloir modifier les comportements en matière d'environnement, qu'il partage avec les tenants de l'approche positiviste, le PFIE est porteur d'un fort désir de changement, voire de rupture par rapport aux orientations et 
aux pratiques d'ERE antérieures, en particulier sur le plan pédagogique. Le renouvellement de la pratique pédagogique des enseignants, que l'on souhaite voir délaisser la pédagogie traditionnelle au profit de la pédagogie active, le désir de former les élèves à partir des réalités locales et l'ouverture de l'école sur le milieu sont d'ailleurs explicitement ciblés comme résultats attendus de ce projet (PFIE/Sénégal, 1999, p. 1-2). Comme nous le verrons plus loin, ces ambitions auront toutefois beaucoup de mal à s'incarner dans la réalité des écoles.

17 La stratégie retenue pour la coordination et la mise en œuvre du projet porte des ambitions similaires de rupture avec les pratiques habituelles, cette fois en matière de planification et de gestion de l'éducation. Cette stratégie est présentée comme une approche créative, associative et active, centrée sur la recherche-action, l'établissement d'un réseau de partenaires, la responsabilisation des acteurs à la base, une approche innovatrice sur le plan pédagogique, des apprentissages réels et utiles et une réflexion productive de solutions pratiques. On retrouve dans cette description plusieurs caractéristiques apparentées au paradigme sociocritique ou à la praxéologie qui y est généralement associée.

\section{Une décentralisation et une recherche-action toutes relatives}

Une analyse plus approfondie montre que l'opérationnalisation de la stratégie adoptée donne lieu à des écarts importants par rapport à la rhétorique avancée. Un coup d'œil sur l'organigramme du projet donne à voir une structure pyramidale, au sein de laquelle on ne voit aucune trace de la participation du personnel enseignant $d u$ primaire, non seulement au niveau des structures responsables du pilotage et de la gestion au plan national, mais également à celui des structures pédagogiques, nationale et locales, qui se partagent la responsabilité de la sensibilisation, de la formation, de l'élaboration des outils pédagogiques, de l'évaluation des apprentissages et du suiviappui. Les pédagogues qui font partie de l'équipe pédagogique nationale proviennent essentiellement des établissements d'enseignement supérieur, d'instituts de recherche ou du ministère de l'Éducation. Quant à ceux qui sont membres des équipes pédagogiques locales, il s'agit exclusivement d'inspecteurs de l'enseignement. Le programme d'éducation environnementale ainsi que les manuels scolaires et les dossiers d'accompagnement élaborés dans le cadre du PFIE portent tous la signature d'inspecteurs et/ou d'universitaires. De ce point de vue, la décentralisation annoncée n'est pas très évidente. Quant à la responsabilisation des acteurs à la base, l'analyse de l'organigramme nous amène à penser que, formellement du moins, son rayon d'action ne déborde pas l'enceinte de l'école.

19 Dans le même ordre d'idées, qualifier la démarche d'expérimentation du PFIE de « recherche-action » paraît quelque peu abusif, dans la mesure où elle n'en partage pas plusieurs des caractéristiques essentielles (Dolbec, 2003, p. 528-529). Certes, à la lumière des rapports d'évaluation (Lelong et Ngom, 1999 ; PFIE/Sénégal, 1999 et 2000 ; Ngom et al., 2001), on ne peut nier son souci élevé d'allier action et réflexion, pas plus que la contribution des enseignantes et enseignants à l'amélioration des pratiques et des outils pédagogiques élaborés. Par contre, on ne saurait considérer ces derniers comme des cochercheurs ou coauteurs, tant la division des tâches est nette entre, d'une part, ceux qui planifient, conçoivent et évaluent l'expérimentation et, d'autre part, ceux qui y participent. Aussi, dans le cas des évaluations d'impact, il paraît difficile d'affirmer que le problème, les objectifs et les méthodes de recherche émergent du processus lui- 
même, alors que l'équipe sénégalaise doit appliquer, avec une marge de manœuvre relativement restreinte, un cadre d'évaluation régional harmonisé et des instruments standardisés (tests d'acquisition, échelles d'attitudes, grilles d'observation, grilles d'évaluation des leçons, questionnaires).

\section{Un programme et un manuel d'éducation environnementale riches, malgré certaines limites}

\section{Une proposition d'ERE ample et bien enracinée dans la réalité du pays}

20 Une analyse du programme d'éducation environnementale et du manuel scolaire du $3 e$ cycle du primaire produits par le PFIE donne à voir une proposition d'ERE relativement ample. Les objectifs retenus font référence aux cinq axes de la proposition de Tbilissi (prise de conscience, connaissances, état d'esprit, compétences et participation à la résolution des problèmes). Le programme (PFIE/Sénégal, 1998a) est structuré autour de trois «compétences » de base: découvrir les problèmes d'environnement et leur impact ; participer à la résolution des problèmes d'environnement; évaluer les actions et leur impact sur l'environnement et la qualité de vie. L'environnement y apparaît avant tout comme un problème à résoudre, le programme étant construit autour de dix-neuf problèmes environnementaux. Par ailleurs, les représentations de l'environnement ressource et de l'environnement milieu de vie sont également présentes, puisque la gestion des ressources naturelles et l'amélioration du cadre de vie sont deux des quatre domaines d'études du programme. Le fait que la participation à la résolution de problèmes environnementaux soit l'une des trois compétences de base du programme donne à penser que l'environnement y est aussi perçu comme une communauté dans laquelle s'engager. Dans ce programme, on trouve très peu de traces d'un environnement comme nature à apprécier, comme système à comprendre pour décider ou comme biosphère où vivre ensemble.

21 L'éventail des problèmes environnementaux retenus reflète une forte prise en compte de la situation environnementale du pays et de ses différentes zones écogéographiques. Pour l'essentiel, les problèmes traités correspondent à ceux qui sont identifiés dans le Plan national d'action pour l'environnement du Sénégal (République du Sénégal MEPN, 1997). Sous cet angle, le programme paraît bien enraciné et signifiant eu égard au contexte. L'affirmation faite dans la préface, selon laquelle le PFIE aurait rompu avec une approche étroite de l'environnement, réduit à la sécheresse et à la désertification, pour s'ouvrir à la pluralité de la réalité sénégalaise et aux problématiques environnementales actuelles, se confirme à l'analyse du programme. En effet, si les zones arides font toujours l'objet d'un traitement particulier, une attention est aussi accordée à d'autres milieux, notamment aux milieux marins et côtiers. De même, le programme déborde le cadre des problèmes environnementaux rencontrés en milieu rural pour s'ouvrir à ceux du milieu urbain. Il va au-delà de l'environnement biophysique pour aborder les problèmes humains (pauvreté, population, santé, patrimoine culturel).

\section{Une vision plutôt restreinte des problèmes, des compétences et des savoirs}

La notion de "problème " paraît abordée dans un sens assez restrictif. Les termes choisis pour désigner les problèmes environnementaux (par exemple, déficit, 
diminution, dégradation, menace, déséquilibre, faiblesse, mauvaise gestion, envahissement, exploitation abusive, extension anarchique) font référence, non pas à une question environnementale complexe à résoudre, mais plutôt à une situation ou à une pratique dommageable pour l'environnement qui est, par extension, condamnable et à combattre. Cette optique colore l'ensemble du programme. Sur le plan des " compétences » que l'on cherche à développer chez l'élève, la reconnaissance des situations de dégradation de l'environnement et la nécessité de rompre avec des pratiques qui y contribuent prennent nettement le pas sur l'exploration des potentialités écosystémiques et humaines du pays et leur mise en valeur.

Le manuel (PFIE/Sénégal, 1998b) fait toutefois contrepoids à cette tendance puisque, en plus d'illustrer abondamment les pratiques de dégradation et leurs conséquences (abattage des arbres, feux de brousse, prélèvement du sable des plages pour la construction, gaspillage de l'eau, pêche à la dynamite ou avec des filets à petites mailles, braconnage, grossesses précoces et naissances rapprochées, exode rural, occupations irrégulières des terrains, consommation de drogue, baignade dans le marigot, nourriture exposée aux mouches), il fait une large place à l'exploration des pratiques, des techniques et des modes de gestion compatibles avec la préservation de l'environnement et l'amélioration de la qualité de vie (reboisement, entretien des ouvrages de protection des terres ou du littoral, mesures d'économie d'eau et d'énergie, classement de forêts, moustiquaires de lit, planning familial, mesures d'hygiène et de prévention, purification de l'eau, fabrication de compost, solidarité). En outre, le manuel témoigne d'un souci d'offrir aux élèves des situations d'apprentissage signifiantes, en puisant abondamment à la réalité endogène. Pour plusieurs des problèmes abordés, qui correspondent à des problèmes environnementaux, sociaux et sanitaires cruciaux dans ce pays, des solutions pratiques, à la portée des élèves, sont proposées (méthode de fabrication du compost, techniques de purification de l'eau, mesures d'hygiène et de prévention des maladies, compréhension des factures d'eau et des notices de médicaments), ce qui indique une préoccupation de développer chez les élèves des compétences utiles dans la vie de tous les jours.

Par ailleurs, une analyse plus approfondie du programme donne à voir une vision plutôt restreinte des savoirs (associés, pour une bonne part, à la capacité de nommer l'existant), des savoir-faire (associés à la capacité d'appliquer des techniques ou de s'engager dans des actions selon des modèles de participation prédéterminés) et des savoir-être (associés à l'évaluation des comportements et des pratiques nuisibles à l'environnement et à la qualité de vie, à l'exclusion des siennes propres), qui vient limiter considérablement sa capacité à produire le changement anticipé en matière d'enseignement et d'apprentissage.

\section{Une vision technologique de l'éducation}

L'analyse du programme d'éducation environnementale permet également de situer le PFIE par rapport aux quatre principales visions paradigmatiques de l'éducation. Le fait que les compétences retenues se calquent sur la démarche de résolution de problèmes de même que l'importance accordée à l'application de techniques amène à associer d'abord le PFIE à une vision technologique de l'éducation. Par ailleurs, l'attention accordée à l'impact des problèmes d'environnement sur la qualité de vie (première " compétence ») et à l'impact des actions humaines sur l'environnement (troisième " compétence ») ainsi que l'accent mis sur la participation (deuxième « compétence ») 
suggèrent que certains éléments d'une vision symbiosynergique de l'éducation ont pu aussi guider la conception de ce programme. Cette vision est toutefois relativement restreinte puisque le fait d'aborder l'environnement biophysique et l'action humaine comme deux entités distinctes qui ont un impact l'une sur l'autre est loin de rendre, dans toute son ampleur, l'interrelation sujet-milieu portée par le paradigme symbiosynergique. De même, si l'objectif d'un agir pertinent traverse le programme, la construction critique de la connaissance connait, pour sa part, un traitement que l'on pourrait qualifier de superficiel. Même si des apprentissages de nature cognitive sont visés, en particulier en ce qui concerne les savoirs rattachés à la première compétence, on trouve peu de traces d'une vision culturaliste de l'éducation, l'accent étant plutôt mis sur la découverte et la compréhension des problèmes que sur l'appropriation de données factuelles et de concepts ${ }^{4}$. Quant à la vision humaniste, centrée sur le sujet qui apprend, elle est pratiquement absente du programme. L'attention accordée à l'élève paraît avant tout motivée par le désir de le préparer à jouer pleinement le triple rôle qu'on lui a assigné en matière d'environnement - celui de témoin avisé, d'acteur engagé et de vecteur d'opinion -, plutôt que par la préoccupation d'assurer son développement intégral.

\section{Un accent marqué sur l'éducation civique, morale et sanitaire}

L'analyse du manuel scolaire du $3^{e}$ cycle du primaire permet d'avoir une idée plus précise des pratiques d'ERE promues par le PFIE, notamment sous l'angle des dimensions éducationnelles et des approches pédagogiques privilégiées. Le manuel propose des apprentissages qui touchent plusieurs dimensions de la formation fondamentale.

L'éducation civique (développement d'un sentiment de responsabilité à l'égard de l'environnement comme patrimoine collectif) et l'éducation morale (développement du sens des responsabilités et de la solidarité) y occupent une place de choix, traversant l'ensemble des sujets abordés. En ce qui a trait à l'éducation morale, il serait toutefois abusif d'évoquer une contribution substantielle au développement d'une réelle compétence éthique, tant l'inculcation de valeurs prend le pas sur l'analyse, la délibération et l'exercice du jugement. La contribution, non négligeable, à l'éducation scientifique et technologique passe principalement par une présentation des concepts et la transmission de données factuelles. Les situations proposées ne semblent pas souvent propices à développer les habiletés à repérer et à évaluer l'information scientifique. Le réinvestissement dans la résolution des problèmes environnementaux est toutefois facilité par l'apprentissage de diverses techniques et méthodes éprouvées. Environ le tiers des leçons est consacré aux apprentissages relatifs à la gestion des ressources, considérée à la fois sous l'angle d'un patrimoine collectif à protéger et d'une consommation individuelle à contrôler, ce qui confère à l'éducation économique une place centrale sur le plan des contenus traités. Un nombre moins important de leçons abordent spécifiquement les problèmes de santé, mais les nombreuses références, tout au long du manuel, à l'impact de la dégradation de l'environnement sur la santé des populations font en sorte que l'éducation à la santé ressort comme une préoccupation majeure. D'autres dimensions sont couvertes, mais de manière plus marginale: l'éducation politique (connaissance de certaines institutions et de leur fonctionnement, mais sans analyse critique), l'éducation sociale (engagement communautaire et coopération dans l'action) et l'éducation personnelle (pouvoir-faire 
et savoir-faire à l'égard de l'amélioration des liens personne-environnement). Seule la contribution à l'éducation esthétique paraît absente.

\section{Un processus d'apprentissage qui privilégie l'approche holistique}

L'analyse du manuel permet aussi d'avoir une idée des approches pédagogiques privilégiées par le PFIE. En ce qui a trait aux approches relatives au processus d'apprentissage, on peut avancer que l'approche holistique est privilégiée, ainsi que les approches systémique et interdisciplinaire qui y sont associées. D'une part, malgré l'accent mis sur la dimension morale, l'élève est considéré dans sa multidimensionnalité. D'autre part, l'environnement est abordé dans sa globalité. Les situations d'apprentissage proposées accordent une place importante aux relations entre les aspects biophysiques et les aspects sociaux des phénomènes, même si celles-ci sont le plus souvent abordées en termes d'impact des uns sur les autres plutôt qu'en termes d'interrelations, comme le voudrait l'approche systémique. Plusieurs préoccupations, par exemple celles ayant trait à l'éducation à la santé, à l'éducation civique et à l'éducation morale, traversent les thématiques traitées, ouvrant la voie à l'interdisciplinarité pédagogique. L'approche critique paraît cependant négligée. Si l'élève est parfois sollicité pour proposer des explications ou des solutions alternatives, il est rarement invité à confronter les valeurs qui lui sont proposées et les siennes propres ou à faire preuve d'un scepticisme réflexif. L'approche résolutique, qui sert de trame au programme, connaît un traitement relativement limité dans le manuel. Mis à part dans les sections explicitement identifiées comme des exercices, l'élève n'est pas amené à s'inscrire dans une réelle démarche de résolution de problèmes, le problème comme la solution lui étant le plus souvent donnés d'avance. Même si des situations de la vie quotidienne sont utilisées comme supports aux apprentissages, on ne trouve pas d'indice d'une réelle approche expérientielle, selon laquelle les apprentissages de l'élève découleraient de son contact direct avec des situations de vie, d'une observation réflexive et d'une expérimentation active sur le terrain. Dans le même ordre d'idées, si l'enquête auprès de membres de la communauté et l'action collective font partie des moyens proposés à l'élève, il serait abusif de dire qu'une place importante est faite à l'approche coopérative, selon laquelle on apprend les uns avec les autres et les uns des autres.

\section{Une approche béhavioriste de l'objet d'apprentissage}

En ce qui concerne les approches adoptées relativement à l'objet d'apprentissage, l'approche béhavioriste paraît dominante. En effet, la plupart des situations ou des actions étudiées sont montrées comme bonnes ou mauvaises et le recours fréquent à l'approbation ou à la désapprobation sociale paraît privilégié comme mode de renforcement des comportements. L'approche affective vient en appui à cette première approche : la peur (de la famine, de la maladie, du rejet social et de la mort) est utilisée pour amener l'élève à rejeter une pratique, alors que la fierté, la confiance en soi et l'esprit de solidarité sont suscités pour stimuler chez l'élève le désir d'adopter de bonnes pratiques et de s'engager dans des actions de changement. Si la référence aux valeurs est très présente, l'approche est plus moralisatrice que morale ou spiritualiste au sens où l'intention paraît être d'inculquer des valeurs à l'élève plutôt que de l'outiller pour qu'il soit capable d'analyser, de clarifier, de confronter les valeurs en jeu et de faire ses propres choix au plan éthique. L'approche cognitive et l'approche 
pragmatique se retrouvent souvent en tandem. Les savoirs d'ordre cognitif, généralement centrés sur la présentation de données factuelles, sont utilisés pour décrire le problème. Ils servent d'introduction à l'appropriation par l'élève de solutions, le plus souvent à caractère technique (qui comportent aussi leur part de cognitif), en vue de la résolution d'un problème environnemental ou social. Malgré son intérêt pour la résolution de problèmes pratiques, on ne saurait toutefois qualifier l'approche de praxique, car l'élève est invité à appliquer des techniques éprouvées plutôt qu'à s'engager dans des projets au sein desquels sa réflexion et son action s'alimenteraient mutuellement.

\section{Une dimension critique qui a du mal à s'incarner dans l'organisation et la pratique pédagogiques}

L'analyse des entretiens et des observations en classe montre que, suite à son appropriation par les acteurs de l'enseignement primaire sénégalais, l'éducation relative à l'environnement portée par le PFIE a connu un rétrécissement certain. Comme nous le verrons, le rétrécissement paraît plus évident encore dans les pratiques effectives (telles qu'elles sont perçues par les observations) que dans les pratiques déclarées (dans le cadre des entretiens).

\section{Une représentation de l'environnement réduite aux problèmes d'insalubrité et d'hygiène}

31 Au regard des finalités de l'ERE, les entretiens avec les intervenants scolaires qui ont participé à l'expérimentation du PFIE laissent voir un accent très prononcé sur le développement de réflexes comportementaux et, dans une moindre mesure, sur la participation à la résolution de problèmes environnementaux. De même, en ce qui concerne les représentations de l'ERE, on ne retrouve pas chez les intervenants scolaires la diversité de la proposition initiale du PFIE. La représentation de «l'environnement ressource » est pratiquement absente de leur discours alors que les pratiques observées la montrent sous un angle très étroit. C'est au regard des problèmes environnementaux abordés que le PFIE semble avoir le plus perdu en richesse. L'environnement que donnent à voir les intervenants scolaires se résume, pour l'essentiel, aux problèmes d'insalubrité et d'hygiène de l'environnement immédiat (l'école, la maison, le quartier), alors que le programme d'éducation environnementale du PFIE couvrait un vaste éventail de problèmes de l'environnement biophysique et humain concernant à la fois la spécificité du milieu sahélien, la gestion des ressources naturelles, les questions de population et l'amélioration du cadre de vie. La préoccupation pour la préservation à plus long terme de l'environnement, et notamment pour la gestion des ressources, semble avoir cédé la place au souci de la résolution immédiate des problèmes d'insalubrité et d'hygiène.

\section{Une vision techno-culturaliste de l'éducation}

En ce qui a trait aux représentations de l'éducation, les personnes interviewées semblent partager la vision technologique portée par le PFIE et voir dans l'appropriation de diverses démarches (résolution de problèmes, projet d'action éducative, approche par compétences) une occasion de valoriser leur acte professionnel, et par là leur profession, en lui conférant un caractère technique qui 
n'est pas à la portée de tous. Cette situation doit aussi être mise en relation avec la formation initiale reçue par la majorité de ces personnes dans les écoles de formation d'instituteurs ou les écoles normales de même qu'avec les pratiques d'évaluation ou d'inspection qui s'intéressent davantage à la capacité du personnel enseignant de reproduire une démarche pédagogique préétablie qu'à son habileté à soutenir les élèves dans leur cheminement d'apprentissage.

\section{Un enracinement assez limité de la proposition initiale du PFIE}

Sur le plan des pratiques d'ERE, l'appropriation de la proposition du PFIE par le personnel enseignant et, plus encore, son enracinement dans l'organisation éducative paraissent, dans l'ensemble, assez limités. Dans la plupart des milieux, on a pu constater un abandon de plusieurs des voies d'intégration de l'ERE à l'école (projet d'action environnementale, visite de sites, jardin scolaire) et un repli sur l'intégration de l'ERE dans l'enseignement des disciplines. Même cette dernière est parfois mise en veilleuse au profit d'un enseignement centré sur la préparation plus directe des élèves aux examens qui, rappelons-le, ne tiennent pas compte de l'ERE.

Même si les intervenants des écoles sont nombreux à se réclamer de la «pédagogie active " portée par le PFIE, l'appropriation des approches pédagogiques du PFIE paraît limitée. En ce qui a trait aux approches relatives au processus d'apprentissage, l'approche holistique, qui n'était pas pleinement déployée dans la proposition initiale, est devenue l'ombre d'elle-même. L'approche interdisciplinaire et l'approche résolutique paraissent un peu plus ancrées, du moins dans les pratiques déclarées, mais elles semblent s'incarner plutôt dans la planification de l'enseignement que dans la pratique de classe. Concernant les approches relatives à l'objet d'apprentissage, on peut noter, comme dans le programme d'éducation environnementale du PFIE, une tendance à privilégier les approches béhavioriste, moralisatrice et pragmatique, mais, cette fois, l'approche cognitive est ignorée.

\section{Des leçons d'ERE centrées sur la modification des comportements des élèves en matière d'hygiène}

L'analyse des observations d'une « leçon d'ERE », réalisées en classe de CP (2e année) et de CM1 (5e année) confirme cette tendance à la réduction de la proposition d'ERE du PFIE dans la pratique de classe. Le tableau 1 permet de saisir l'écart qui sépare l'ERE initialement proposée par le PFIE de celle qui a cours sur le terrain. 
Tableau 1 : Mise en relation de la proposition d'ERE du PFIE sénégalais avec ce qui se dégage de l'analyse des entretiens dans les écoles et des observations en classe

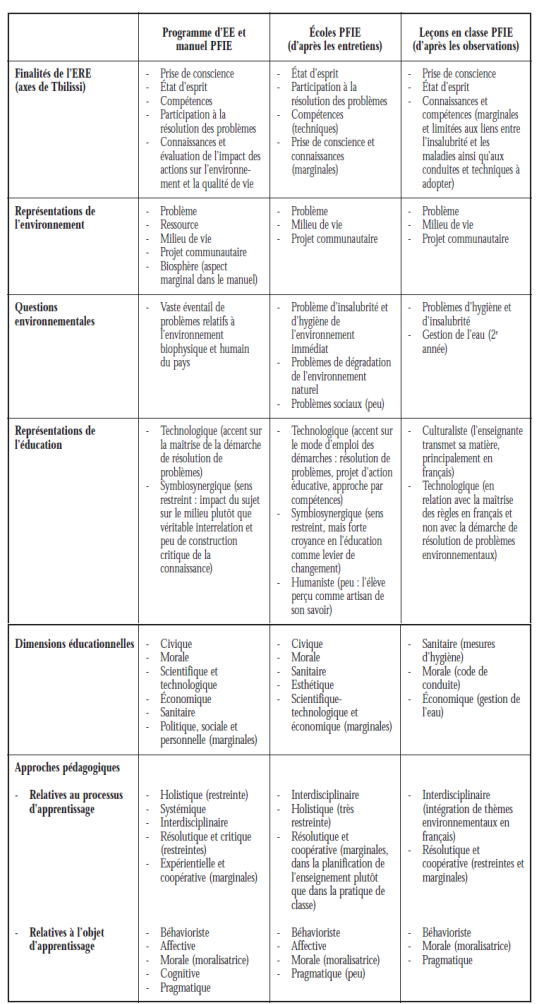

Sur le plan pédagogique, il est difficile de voir les leçons observées comme des exemples de pédagogie active, courant dont les enseignantes et enseignants qui ont participé au PFIE se réclament. Le travail des élèves se limite, pour l'essentiel, à appliquer les consignes de l'enseignante et à lui fournir « la » bonne réponse. Le travail individuel demandé à l'élève fait peu appel à son autonomie et à sa créativité (répétition à l'oral ou écriture sur ardoise de la bonne réponse). Les élèves ont très peu l'occasion de participer, malgré l'intérêt qu'ils démontrent en levant la main et en claquant des doigts pour répondre aux questions. Ils passent beaucoup de temps à écouter la même réponse fournie à chaque fois par une dizaine d'élèves. Les temps morts sont très nombreux. De plus, il n'y a aucune interaction entre élèves, aucune délibération ou confrontation des idées, des démarches ou des valeurs. La seule personne réellement active sur le plan pédagogique semble être l'enseignante. Elle est fort occupée à soutenir l'intérêt des élèves, qui paraissent par ailleurs excessivement motivés, à sélectionner ceux qui vont répondre à ses appels en se souciant que le plus grand nombre participe, y compris les plus faibles, à corriger les erreurs de prononciation ou d'orthographe sans humilier l'élève fautif, et à rappeler à l'ordre ceux qui parlent sans autorisation ou qui rient d'un élève moins performant (comportements peu fréquents), le tout, avec le souci constant de "passer sa matière ». Son rôle central, voire monopolistique, est accentué par l'absence d'utilisation de manuel scolaire ou de tout autre support pédagogique, à l'exclusion du tableau noir, des ardoises et de quelques objets pour un jeu de rôle.

Sur le plan des buts de l'ERE, ces « leçons " s'intéressent principalement à la prise de conscience des problèmes d'environnement et à la modification des comportements en la matière. Le développement de "compétences" se limite à l'apprentissage de mesures simples d'hygiène, de traitement des déchets ou d'économie d'eau, et ce, tant 
dans la classe de 5e année que dans celle de 2e année. L'environnement y apparaît comme un problème d'hygiène à résoudre et un milieu de vie à améliorer. La vision culturaliste de l'éducation semble guider la pratique des enseignantes, préoccupées de " passer leur matière ». On peut supposer que les conditions concrètes de la pratique de l'enseignement (groupes pléthoriques pouvant atteindre 100 élèves, carence de matériel pédagogique) contribuent au repli sur des pratiques d'enseignement magistral. Par ailleurs, comme certains répondants l'ont souligné, la tendance des maîtres à enseigner comme ils ont été formés, c'est-à-dire selon une approche de transmission de connaissances, pourrait tout autant, sinon plus encore, expliquer cette situation.

Les dimensions éducationnelles touchées sont l'éducation sanitaire (apprentissage des mesures d'hygiène), l'éducation morale (apprentissage des codes de conduite) et, dans une moindre mesure, l'éducation économique (gestion des ressources en eau). En ce qui a trait aux approches pédagogiques relatives au processus d'apprentissage, l'interdisciplinarité est privilégiée. Elle prend la voie de l'utilisation de thèmes environnementaux comme support aux apprentissages en français et en éducation sanitaire. On est cependant très loin d'un réel croisement des savoirs, notamment parce que l'ERE reste en périphérie de l'action pédagogique, voire de l'intention pédagogique, sauf en ce qui a trait au développement de certains savoir-être et savoir-faire d'un niveau de complexité très bas. En ce qui concerne l'objet d'apprentissage, les approches béhavioriste, moralisatrice et pragmatique semblent inspirer les enseignantes.

Les entretiens et les observations en classe suggèrent que les représentations «traditionnelles » de l'école et de l'enseignement, dont les enseignantes et enseignants prétendent s'être distanciés, n'ont pas véritablement été délogées et continuent d'inspirer leurs pratiques. Une certaine sédimentation semble s'opérer, les emprunts aux nouvelles représentations s'ajoutant aux représentations antérieures. Il semble que le désir des enseignantes et enseignants d'adopter la " pédagogie active » ait du mal à s'ancrer tant au niveau des représentations qui guident leurs pratiques qu'au niveau des pratiques elles-mêmes. À cet égard, il faut rappeler qu'ils doivent composer avec des messages contradictoires en provenance des instances centrales et départementales du ministère de l'Éducation. D'une part, ils sont incités à poursuivre l'implantation du PFIE, qui porte cette aspiration de renouvellement pédagogique, pendant que, d'autre part, ils sont tenus de respecter les prescriptions du décret $\mathrm{n}^{\mathrm{o}}$ 79-1165 qui, dans son esprit comme dans sa lettre, n'a rien de la pédagogie active. Il faut savoir que le décret $\mathrm{n}^{\circ}$ 79-1165 (République du Sénégal, 1979), qui organise l'enseignement élémentaire sénégalais, fixe, pour chaque degré de scolarité, les disciplines prévues et, pour chacune d'elles, les objectifs, les contenus précis de formation présentés selon leur progression chronologique, ainsi que l'horaire et la programmation détaillés (par exemple, $1 \mathrm{~h} \mathrm{15}$, répartie en cinq séances de quinze minutes, dont trois d'écriture et deux de copie). On y précise de plus l'objet des leçons (par exemple, première journée : tracé de lettres ou copie de mots étudiés en lecture), le médium utilisé (tracé sur l'ardoise), le nombre de mots ou la longueur des phrases, la fréquence des exercices de contrôle et des devoirs, et jusqu'au type de mine de crayon acceptable.

On comprendra que les contraintes de système qui s'exercent sur un projet comme celui du PFIE sont telles que la rupture annoncée avec le paradigme dominant tout 
comme les changements pédagogiques et environnementaux attendus ne peuvent avoir, dans leur traduction concrète, le caractère radical qui leur est attribué.

\section{Des signes d'une ERE critique sous le conformisme apparent}

41 La caractérisation critique de la proposition d'ERE du PFIE sénégalais présentée dans les pages qui précèdent - la proposition initiale et, plus encore, ce qu'elle est devenue sur le terrain - peut en donner l'image d'une ERE très limitée et conservatrice, tant sur le plan environnemental que pédagogique. Pourtant, sous ce conformisme apparent, se cachent des signes d'une ERE critique qu'il importe de débusquer, en tentant de saisir le sens que revêtent les phénomènes observés dans le contexte particulier de l'enseignement primaire sénégalais. Pour cela, il faut s'intéresser à la rupture que cette ERE représente par rapport aux représentations et aux pratiques dominantes plutôt qu'à la distance qui la sépare des orientations internationales, y compris celles du courant critique, le plus souvent "occidentalement» définies. Un regard moins normatif et plus attentif au contexte montre que, en ce qui concerne les représentations et les pratiques en matière d'éducation, d'environnement et d'ERE, le PFIE a contribué, sinon à une rupture, du moins à un renouvellement certain.

\section{Une autonomie qui sort des sentiers battus}

Certes, comme cela a été souligné précédemment, ce projet était loin d'incarner l'idéal de décentralisation et de recherche-action, tel que nous le concevons dans les pays du Nord. Par ailleurs, il faut reconnaître que, dans un système fortement centralisé et hiérarchisé comme le système éducatif sénégalais, le fait que les inspecteurs départementaux s'engagent dans des partenariats sans requérir une approbation ministérielle, qu'une école adopte son propre plan d'action en matière d'éducation environnementale sans contrôle de l'inspecteur, ou encore, que le personnel enseignant puisse ajuster la grille horaire fixée par décret, représente une manifestation certaine de décentralisation-responsabilisation.

\section{Un outil privilégié de changement social}

Les acteurs du PFIE sénégalais que nous avons interviewés se définissent d'ailleurs comme des agents de changement. Pour eux, l'éducation est un outil puissant de changement social, notamment parce que l'enfant-élève est vecteur d'informations, d'opinions et de pratiques nouvelles au sein de sa famille et de son quartier. Dans le cadre des entretiens, les références aux contre-valeurs de la rue, auxquelles sont confrontés les enfants dans l'adoption de nouveaux comportements, de même que l'évocation de la résistance sociale à laquelle les intervenants scolaires doivent euxmêmes faire face dans l'implantation de nouvelles approches éducatives, témoignent de cette représentation de l'éducation, et plus spécifiquement de l'ERE, comme levier de changement. Les altercations de certains enseignants avec des parents, qui finissent par entendre raison, sont racontées comme des faits d'armes qui confirment à la fois la difficulté et la justesse de l'intervention du personnel enseignant et, plus largement, de son rôle comme acteur du changement. Dans le même ordre d'idées, les personnes interviewées présentent l'éducation, et notamment l'ERE, comme un processus qui agit 
en profondeur et sur le long terme, ce qui, à leurs yeux, lui donne une valeur supérieure aux formes d'activisme promues par des activités comme les vacances citoyennes. Plusieurs se montrent préoccupés d'une plus grande équité sociale, exprimant leur rejet d'un système d'écoles à deux vitesses et leurs attentes à l'effet que l'ERE contribue au développement de compétences de la vie courante et à l'insertion sociale des jeunes.

\section{Une occasion de valoriser la profession enseignante}

On peut se demander s'il n'y a pas là un signe d'une mutation progressive du rôle de l'école et du personnel enseignant dans les pays en développement. En effet, l'éducation, auparavant considérée comme le moyen de préparer les élites, est désormais vue comme l'un des piliers du développement et de la lutte contre la pauvreté. L'instituteur, dont le statut de détenteur du savoir l'amenait à agir comme interface entre les populations et le monde lettré, incluant l'administration, se voit progressivement confier le rôle de médiateur entre l'école et son environnement social. Le personnel enseignant semble trouver dans ce rôle d'éveilleur de conscience et de passeur, qu'il associe à une "mission noble ", une source de motivation. Compte tenu de l'insatisfaction importante vécue par le corps enseignant sénégalais, dont environ les trois quarts souhaiteraient changer de carrière (Chinapah, 1999), cette contribution à la redynamisation du personnel enseignant peut être considérée comme un apport non négligeable du PFIE.

De la même manière, la dominance observée de la vision technologique de l'éducation chez les acteurs du PFIE mais plus particulièrement chez le personnel enseignant, n'est peut-être pas sans lien avec le désir de valoriser la profession enseignante. Contrairement à la situation qui prévaut au Québec (Conseil supérieur de l'éducation, 1991), en contexte sénégalais, la mise en évidence de la technicité de l'intervention pédagogique peut être vue comme une stratégie, consciente ou non, de valorisation de la profession enseignante. La technique étant revêtue d'une auréole de modernité, l'accoler à l'acte d'enseigner contribue à en rehausser la valeur et la reconnaissance sociale, en particulier dans un pays en développement où les aspirations à la modernité sont élevées. L'insistance sur le caractère systématique et complexe des processus pédagogiques dénote aussi, à notre avis, un désir de distanciation par rapport à une représentation de l'acte d'enseigner vu comme un bricolage artisanal à la portée de tous. Cette représentation, dans laquelle la science et la technique paraissent supérieures au sens commun, fortement apparentée à l'idéologie scientiste, peut aussi être, pour les enseignantes et enseignants, une manière de réagir à la restructuration en cours de leur profession, et notamment au recrutement de volontaires de l'éducation, sans formation pédagogique initiale, auquel l'État sénégalais a amplement recours pour réduire les coûts associés à l'augmentation de l'effectif enseignant découlant de la massification de la scolarisation primaire.

\section{Un souci de répondre aux besoins urgents du milieu}

En ce qui a trait à l'accent mis sur les dimensions sanitaire et morale de l'ERE qui, de prime abord, donne l'impression d'une certaine étroitesse des représentations et des pratiques d'ERE des acteurs du PFIE, on en saisit mieux le sens lorsqu'on le met en relation avec la situation du pays. Le Sénégal se classe au 157e rang mondial sur 177 
pays en ce qui a trait à l'indicateur de développement humain du Programme des Nations Unies pour le développement (PNUD, 2005). L'espérance de vie à la naissance y est de 52,7 ans. Le paludisme de même que les infections gastro-intestinales y font chaque année des milliers de victimes, en particulier chez les enfants. Dans un tel contexte, les perspectives d'avenir se résument parfois à assurer la survie du plus grand nombre jusqu'au lendemain. On comprend dès lors l'urgence de la situation qui amène les intervenants pédagogiques à chercher une modification immédiate des comportements en matière d'hygiène, de salubrité et d'amélioration du cadre de vie plutôt qu'une formation plus ample à la complexité des situations environnementales. De la même manière, si les incantations moralisatrices et la stigmatisation des mauvais comportements peuvent agacer, on comprend néanmoins le désir de développer une éthique personnelle et un comportement socialement responsable qui les sous-tend, sachant que le pays est miné par le clientélisme et la corruption (Diop, 2002 et Transparency International, 2005). Sans nier que cette très forte centration sur le présent et sur le développement de "réflexes comportementaux", qui a comme corollaire la faible attention accordée au développement des capacités cognitives des élèves, peut compromettre la contribution de l'ERE aux changements éducatifs et environnementaux requis à plus long terme, il faut reconnaître qu'elle témoigne d'un enracinement dans les besoins locaux.

Bon nombre de répondantes et répondants se montrent à la fois soucieux et satisfaits de l'utilité des réalisations du PFIE en matière environnementale. Les résultats visibles dans l'environnement de proximité font d'ailleurs partie des raisons majeures pour lesquelles ils tracent un bilan positif du PFIE. Les changements survenus dans l'environnement de l'école et du quartier (entretien, embellissement, culture et vente de fruits comestibles, respect de l'arbre et culture de la propreté, aménagement des cours), imputés au PFIE, sont appréciés avant tout pour leur utilité au regard de l'amélioration du cadre de vie scolaire et des conditions de vie des populations. De la même manière, si l'ouverture à une ERE citoyenne semble assez présente, comme le montre l'accent mis sur la participation et l'engagement des élèves dans leur communauté, le caractère planétaire de cette citoyenneté ne paraît susciter de l'intérêt que chez quelques répondants, les autres se montrant plus préoccupés de répondre aux appels urgents de la vie quotidienne et de l'environnement immédiat. En cela, les propos de plusieurs répondantes et répondants reflètent un rôle pragmatique, voire instrumental de l'ERE. Cette vision témoigne aussi de l'enracinement de l'école dans son milieu et du caractère exemplaire des pratiques qu'on souhaite y développer, rompant ainsi avec une école perçue comme coupée de sa communauté et incapable de développer des compétences utiles dans la vie courante et de préparer à l'insertion socioprofessionnelle.

Dans le même ordre d'idées, l'intérêt manifesté pour l'évolution du champ conceptuel en ERE ou pour l'appropriation de concepts environnementaux par les élèves ne semble pas faire le poids face à l'urgence d'outiller concrètement les élèves pour la résolution des problèmes qu'ils rencontrent dans leur environnement immédiat. De l'avis de l'un des coordonnateurs du programme, les longs débats théoriques et les détours conceptuels font figure de luxe que ne peuvent s'offrir des pays en développement aux prises avec de dures "réalités quotidiennes ». Cette attention aux particularités du contexte africain militerait en faveur d'une ERE qui, au lieu de "changer de concepts " chaque décennie ou tous les cinq ans, travaillerait plutôt « sur la durée pour modifier 
des comportements ", aider les gens à résoudre leurs " problèmes pratiques » en leur apprenant "des petites techniques ». Ces dernières remarques ne sont pas dénuées d'intérêt. Elles reprennent, dans un autre registre, les critiques formulées à l'endroit du caractère décontextualisé des activités de recherche en ERE, y compris celles du courant critique, caractérisées par une tendance à la « linguistification » de la réalité, renforcée par des échanges intellectuels désincarnés entre experts qui survalorisent l'abstrait au détriment de l'expérience sensorielle et de la prise en compte du contexte socio-écologique (Hart, 2005 ; O’Donoghue et Lotz-Sisitka, 2005 ; Payne, 2005).

\section{La conscience de l'incompatibilité entre sous-développement et environnement}

Les critiques des excès du développement - qu'il s'agisse des appels pour stopper la croissance, des invitations à la simplicité volontaire ou de la mobilisation contre les OGM - trouvent peu d'écho chez les acteurs du PFIE sénégalais. On peut penser que ce n'est pas l'ignorance, mais plutôt leur bonne connaissance de la situation environnementale de leur pays, où le sous-développement et la pauvreté alimentent la détérioration de l'environnement, qui contribue à les rendre plus sceptiques face à des slogans importés qui postulent une certaine incompatibilité entre le développement et l'environnement.

50 Les craintes exprimées par les répondantes et répondants quant aux effets potentiellement négatifs du développement portent plutôt sur les risques à l'environnement social que sur ceux liés à l'environnement biophysique, notamment lorsqu'il est question de développement touristique. Celui-ci est à la fois souhaité et vu comme menaçant. Des intervenants font état d'un "problème de tourisme ", des touristes qui les "envahissent", "des maladies qui viennent" et des problèmes sociaux, situation face à laquelle il faut éduquer les enfants. Même si les propos sont empreints d'une certaine pudeur, la crainte d'une extension de la prostitution, y compris juvénile, et du sida qui l'accompagne est manifeste. Loin de la seule frilosité morale à laquelle cette réserve face à l'influence extérieure est parfois associée, on peut y déceler, chez ces personnes, une conscience aiguë des risques auxquels sont exposés les jeunes de leur pays, d'autant plus enclins à céder aux sirènes de la marchandisation des corps et des esprits qu'ils sont fortement fragilisés par la précarité économique. Cette analyse rejoint celle d'Aminata Traoré (2002, p. 180), selon laquelle le tourisme est « ambigu » et de nature à exposer les communautés, l'environnement et l'héritage culturel à des dangers que les pays africains ont souvent du mal à prévenir et endiguer (consommation d'alcool, de tabac ou de drogue influant sur les comportements des jeunes, prostitution des enfants, mendicité, pollution).

\section{Un rejet du néo-colonialisme}

51 Enfin, les propos de certains répondants dénotent une conscience et un rejet du néocolonialisme qui caractérise les projets d'aide au développement, dont le PFIE. Des représentants ministériels déplorent le fait que le Sénégal a "gagé son développement sur des ressources extérieures ", ce qui compromet la pérennisation du PFIE. Un partenaire présente un point de vue convergent, soulignant que « le gros problème des projets ici, dans nos pays, c'est que quand le financement s'arrête, quand le robinet est arrêté, alors il y a une bonne partie des actions qui ne continuent pas». Un promoteur du PFIE fait état d'un autre problème, qui découle de la dépendance financière, celui de 
l'ingérence des bailleurs de fonds qui perturbe les travaux de réforme éducative: chaque bailleur vient avec ses façons de faire, son système et ses experts. Dès que ça change de bailleurs, on repart à zéro, alors que le Sénégal dispose d'experts nationaux, qui ont même été formés à l'étranger.

\section{Conclusion}

Le PFIE, au-delà de sa spécificité, doit être situé dans le double mouvement, pédagogique et environnemental, de renouvellement de l'école sénégalaise. Ses concepteurs, tout comme les acteurs de terrain qui y participaient, portaient l'ambition de rompre avec les pratiques dominantes en éducation: planification centralisée des activités excluant le personnel enseignant, fermeture de l'école par rapport au milieu, pédagogie traditionnelle, absence de prise en compte des objectifs et des contenus relatifs à l'environnement dans les programmes d'études et les activités scolaires.

Sous cet angle, malgré les limites que nous avons constatées (par exemple, absence du personnel enseignant des structures d'orientation et des activités de conception du projet, accent sur la modification des comportements des élèves en matière environnementale, faible complexité des objectifs d'apprentissage), on peut néanmoins avancer que l'apport du PFIE a été substantiel. En lui-même, le travail d'élaboration de la proposition et de gestion du projet a impliqué un effort collectif de conception, de formation, de communication et de partenariat qui, de l'avis unanime des personnes qui y ont été associées, représente une expérience sans précédent de mise à profit des compétences nationales et de renforcement des capacités des acteurs et partenaires de la scène éducative.

54 Toutefois, en référence aux ambitions portées par la Charte de Belgrade (UNESCO/ PNUE, 1976), en particulier en ce qui concerne l'émergence d'un nouvel ordre économique et social, on peut dire que la contribution de la proposition du PFIE était plutôt modeste. Son silence sur les structures de pouvoir (politiques, économiques et sociales) qui créent ou entretiennent les problèmes environnementaux de même que sa propension à reproduire les normes sociales en vigueur en font un projet d'ERE relativement conforme à l'ordre établi. En ce sens, il confirme la dilution de l'orientation initiale de l'ERE déplorée par Barbara Bader (1998-1999 et 2001).

Même si, mesuré à l'aune des textes fondateurs de l'ERE ou des postulats du paradigme socio-critique, le PFIE peut sembler manquer d'envergure, il est clair que, dans son contexte, dont les prescriptions du décret 79-1165 donnent un aperçu, il représentait une véritable révolution. La résistance sociale à laquelle il a donné lieu dans les débuts de son expérimentation - certains parents menaçant de retirer leur enfant de l'école donne un indice de la rupture qu'il opérait avec les traditions pédagogiques existantes.

56 À cet égard, on ne doit pas sous-estimer son potentiel de résistance culturelle et politique. Sa valorisation des productions endogènes, dont le programme d'éducation environnementale et les manuels du PFIE fournissent un exemple, sa promotion de la solidarité africaine et plus spécifiquement sahélienne, dans le fonctionnement même du projet comme dans le programme d'éducation environnementale, son souci de réintroduire les valeurs et savoirs traditionnels aux côtés des savoirs modernes, sa capacité à faire cohabiter la sagesse du doyen et le dynamisme de l'enfant-vecteur sont aussi le signe d'une certaine distance à l'égard de l'occidentalisation du monde et d'une 
réhabilitation des visions du monde africaines qui pourraient servir d'assises à des alternatives d'écodéveloppement, dans la foulée des appels lancés par Serge Latouche (1998) et Aminata Traoré (1999 et 2002).

\section{BIBLIOGRAPHIE}

Bader, B. (1998-1999). Enseignement scientifique, technologique et éducation relative à l'environnement sans recul réflexif : une conjugaison risquée. Éducation relative à l'environnement : Regards - Recherches - Réflexions, 1, 187-194.

Bader, B. (2001). Étude de conversations estudiantines autour d'une controverse entre scientifiques sur la question du réchauffement climatique. Thèse de doctorat, Université Laval.

Berthelot, M. (2005). Recherche participative en éducation relative à l'environnement : quelques questionnements sur le leadership des chercheurs et l'expression des acteurs. In Sauvé, L., Orellana, I. et Van Steenberghe, É. (dir.), Éducation et environnement : un croisement des savoirs (p. 221-225). Montréal : Les Cahiers scientifiques de l'ACFAS (Association francophone pour le savoir) no. 104.

Chinapah, V. (dir.) (1999). Avec l'Afrique pour l'Afrique - Vers l'éducation de qualité pour tous. Projet de rapport régional MLA (« Monitoring Learning Achievement »/Suivi permanent des acquis scolaires). Paris : UNESCO/UNICEF.

Conseil supérieur de l'éducation. (1991). La profession enseignante : vers un renouvellement du contrat social. Québec : Conseil supérieur de l'éducation.

Diop, M.-C. (dir.) (2002). La société sénégalaise entre le local et le global. Paris : Karthala.

Dolbec, A. (2003). La recherche-action. In Gauthier, B. (dir.), Recherche sociale : de la problématique à la collecte de données (p. 505-540). Sainte-Foy : Presses de l'Université du Québec.

Hart, P. (2005). Transitions in thought and practice : links, divergences and contradictions in post-critical inquiry. Environmental Education Research, 11(4), 391-400.

Latouche, S. (1998). L'autre Afrique : entre don et marché. Paris : Albin Michel, Économie.

Lelong, M. et Ngom, E. (1999). Appui méthodologique au PFIE pour les études d'impact et d'évaluation en éducation environnementale - Partie 1 - Cadre méthodologique. Université de Liège et INÉADE de Dakar.

Naudet, J.-D. (1999). Trouver des problèmes aux solutions - Vingt ans d'aide au Sahel. Paris : OCDE.

Ngom, E., Ndiaye, M. et Soukouna, H. (2001). Évaluation de l'utilisation des outils pédagogiques du PFIE. Dakar.

O'Donoghue, R. et Lotz-Sisitka, H. (2005). Towards a better grasp of what matters in view of «the posts ». Environmental Education Research, 11(4), 445-454. 
Payne, P.G. (2005). Lifeworld and textualism : reassembling the researcher/ed and « others ». Environmental Education Research, 11(4), 413-431.

PFIE/Sénégal (1998a). Programme d'éducation environnementale à l'école élémentaire.Dakar : PFIE/Sénégal.

PFIE/Sénégal (1998b). Pour mieux vivre au Sahel - Troisième étape CM1/CM2. Dakar : PFIE/ Sénégal.

PFIE/Sénégal (1999). Éducation environnementale au Sénégal - Acquis et défis. Forum international au Sahel sur l'éducation environnementale. Dakar : PFIE/Sénégal.

PFIE/Sénégal (2000). Évaluation d'impact de l'éducation environnementale - Volet Sénégal. Dakar : PFIE/Sénégal.

PNUD. (2005). Rapport mondial sur le développement humain 2004. Consulté le 15 juillet 2004 sur : http://www.undp.org.

République du Sénégal. (1979). Décret N 79-1165 du 20 décembre 1979 portant organisation de l'Enseignement élémentaire. Dakar : Gouvernement du Sénégal.

République du Sénégal - Ministère de l'Environnement et de la Protection de la nature. (1997). Plan national d'action pour l'environnement. Dakar : Secrétariat permanent du Conseil supérieur des ressources naturelles et de l'environnement.

Robottom, I. et Hart, P. (1993). Research in Environmental Education - Engaging the Debate. Geelong : Deakin University Press.

Sauvé, L. (1997). Pour une éducation relative à l'environnement : éléments de design pédagogique. Montréal : Guérin, $2^{\mathrm{e}}$ édition.

Sauvé, L. et Garnier, C. (2000). Une phénoménographie de l'environnement : réflexions théoriques et méthodologiques sur l'analyse des représentations sociales.In Garnier, C. et Rouquette, M.-L. (dir.), Représentations sociales et éducation (p. 211-232). Montréal : Les Éditions nouvelles.

Smith, S. (2003). Négrologie. Pourquoi l'Afrique meurt ? Paris : Calmann-Lévy.

Transparency International. (2005). Corruption Perceptions Index 2005.Consulté le 5 novembre 2006 sur : http://www.transparency.org.

Traoré, A. (1999). L'étau - L’Afrique dans un monde sans frontières. France : Actes Sud/Babel.

Traoré, A. (2002). Le viol de l'imaginaire. France : Actes Sud/Fayard.

UNESCO/PNUE (1976). La Charte de Belgrade. Connexion, 1(1), 1-3.

UNESCO/PNUE (1978). Rapport final. Conférence intergouvernementale sur l'éducation relative à l'environnement, Tbilissi, URSS, 14-26 octobre 1977. Paris : UNESCO.

\section{NOTES}

1. Les réflexions qui sont livrées dans cet article s'appuient sur une recherche doctorale plus vaste. La thèse sur laquelle elle a débouché, réalisée en 2007 sous la co-direction de Renée Cloutier et Lucie Sauvé, s'intitule Étude de la contribution du Programme de formation-information pour l'environnement à la pérennisation de l'éducation relative à l'environnement dans l'enseignement primaire sénégalais. Elle peut être consultée sur les sites Internet suivants : www.theses.ulaval.ca ; 
www.espace-ressources.uqam.ca/images/Documents/Recherche/Theses/

M.BERTHELOT(2007).pdf

2. Dans la typologie qu'elle a développée, Lucie Sauvé cerne sept principales représentations types de l'environnement: une nature à apprécier, à respecter, à préserver ; une ressource à gérer; un problème à résoudre ; un système à comprendre pour décider ; un milieu de vie à connaître, à aménager; une biosphère où vivre ensemble et à long terme; un projet communautaire où s'engager (Sauvé, 1997 ; Sauvé et Garnier, 2000). Sauvé (1997) cerne trois principales perspectives de l'ERE : une perspective environnementale, selon laquelle l'ERE est d'abord perçue comme un outil de résolution de problèmes et de gestion de l'environnement biophysique; une perspective éducative, selon laquelle l'ERE contribue au développement intégral de la personne et de son groupe social, en relation avec le milieu de vie; une perspective pédagogique, selon laquelle l'ERE est vue comme un mouvement de renouvellement des principes et des pratiques pédagogiques. S'appuyant sur les travaux antérieurs d'Yves Bertrand et de Paul Valois, elle distingue quatre visions paradigmatiques de l'éducation: une vision humaniste, centrée sur le sujet qui apprend; une culturaliste, centrée sur la transmission d'un objet d'apprentissage, le plus souvent de nature cognitive ; une technologique, axée sur la maitrise par l'agent d'une démarche ou d'une technologie particulière; une symbiosynergique, axée sur l'interrelation sujet-milieu en vue d'une construction critique de la connaissance et d'un agir pertinent. Dans le prolongement des travaux de Renald Legendre sur la formation fondamentale, cette auteure souligne la contribution potentielle de l'ERE aux dimensions éducationnelles suivantes : l'éducation personnelle, sociale, morale, scientifique, civique, politique, économique, relative à la santé et esthétique. En ce qui a trait aux approches pédagogiques en ERE, Lucie Sauvé identifie sept approches relatives au processus d'apprentissage (expérientielle, holistique, systémique, interdisciplinaire, coopérative, critique et résolutique) et sept approches relatives à l'objet d'apprentissage (cognitive, affective, pragmatique, morale, spiritualiste, béhavioriste et praxique).

3. 3 Une immersion prolongée dans le milieu de l'éducation au Sénégal a grandement facilité la collecte et l'analyse des données. De 2000 à 2003, je travaillais comme conseillère à la programmation au siège de la Conférence des ministres de l'Éducation des pays ayant le français en partage (CONFEMEN) à Dakar. Cette expérience m'a notamment aidée à mieux saisir les relations entre le contexte et le travail de recherche (Berthelot, 2005).

4. Même si la vision culturaliste de l'éducation est peu présente dans le programme d'éducation environnementale du PFIE, comme nous le verrons plus loin, elle occupe cependant une place importante dans le manuel scolaire et dans les pratiques du personnel enseignant.

\section{RÉSUMÉS}

Cet article présente les résultats d'une étude critique du Programme de formation-information pour l'environnement (PFIE) implanté au Sénégal entre 1990 et 2001. L'analyse des données recueillies fait ressortir que, même si le PFIE n'avait pas toute l'envergure de la proposition d'éducation relative à l'environnement (ERE) définie dans les textes fondateurs de l'UNESCOPNUE et qu'il s'apparentait, sous plusieurs aspects, à une approche positiviste de l'ERE, il constituait néanmoins un enrichissement appréciable en matière d'éducation relative à l'environnement au Sénégal. L'analyse montre toutefois que des écarts importants séparent la proposition formelle du PFIE, telle qu'élaborée par ses concepteurs, de sa traduction sur le 
terrain, au contact des acteurs de première ligne et des contraintes avec lesquelles ils doivent composer. Sans nier les changements qui s'imposent, dont certains acteurs du PFIE font euxmêmes état, cet article aborde également les éléments de résistance et de rupture qui se cachent sous le conformisme apparent et qui nourrissent le processus de transformation de l'ERE au Sénégal.

This article presents the results of a critical study of an environmental education (EE) program (PFIE - Programme de formation-information pour l'environnement) implemented in Senegal between 1990 and 2001. The data analysis shows that even if the Program did not have the full scope of the environmental education proposal stemming from UNESCO-UNEP founding documents and that it was associated, in many aspects, with a positivist EE approach, it nevertheless represented a considerable contribution to EE in Senegal. However, the analysis also shows important variations between the Program's formal proposal, as defined by its authors, and its practical application, where actors must compose with some constraints. Without denying the necessity for certain changes, that some Program actors mention themselves, this article also reflects on elements of resistance and rupture which hide under apparent conformism and contribute to the transformation process of EE in Senegal.

\section{AUTEUR}

\section{MICHĖLE BERTHELOT}

Après un diplôme d'études approfondies en recherches comparatives sur le développement (École des hautes études en sciences sociales, Paris), elle a poursuivi ses études doctorales en administration et évaluation en éducation (Université Laval, co-direction Université du Québec à Montréal). Sa thèse, déposée en 2007, porte sur l'éducation relative à l'environnement dans l'enseignement primaire sénégalais. Elle travaille actuellement comme conseillère en affaires internationales au ministère de l'Éducation, du Loisir et du Sport du Québec. 